\title{
Footprints of functional decline: using complementary physiological and behavioural biomarkers as proxies for population dynamics over space and time
}

\author{
Susanne Shultz ${ }^{1}$, Jake Britnell ${ }^{1}$, and Nicholas Harvey ${ }^{1}$ \\ ${ }^{1}$ University of Manchester
}

June 18, 2020

\begin{abstract}
Linking environmental conditions to the modulators of individual fitness is necessary to predict long-term population viability and resilience. Behavioural and physiological biomarkers can provide this mechanistic insight into how individuals perceive and respond to environmental challenges through primary physiological responses, secondary downstream responses and tertiary whole organism responses. To fully exploit biomarkers, we need to move beyond single biomarker studies to develop an integrative approach that models the interactions between extrinsic challenges, physiological and behavioural pathways and their modulators. Here we introduce two frameworks for using multiple integrated biomarkers to establish changes in functional condition. The Functional Marginality Hypotheses proposes that relative changes in allostatic load, reproductive health and behaviour can evidence and establish causation driving macroecological processes such as local extirpation, colonisation, population dynamics and range change. The Functional Recovery Hypothesis proposes that a similar approach can serve as a valuable conservation tool for evaluating individual and population level health, predicting responses to future environmental change and measuring the impact of interventions. We highlight specific studies that have used complementary biomarkers to link extrinsic challenges to population performance. This framework of integrated biomarkers has untapped potential to identify causes of decline, predict future changes and mitigate against future biodiversity loss.
\end{abstract}

\section{Hosted file}

Footprints of decline_EcolLett_submitted_with figures.pdf available at https://authorea. com/users/334755/articles/460675-footprints-of-functional-decline-using-complementaryphysiological-and-behavioural-biomarkers-as-proxies-for-population-dynamics-over-spaceand-time 